\title{
Performance improvement of synthetic Brillouin optical time-domain reflectometer employing Golay coding
}

\author{
Ken'ichi Nishiguchi \\ Office for University-Industry Collaboration, Osaka University \\ Open Laboratory F-172, ISIR, 8-1, Mihogaoka, Ibaraki, Osaka 567-0047, Japan
}

\begin{abstract}
Recent advances in Brillouin optical time-domain reflectometry (BOTDR) technology have enabled the high resolution distributed fiber optic sensing of strain and temperature. However, a high spatial resolution BOTDR, such as a synthetic BOTDR (S-BOTDR), has a weak point: the spectrum's signal-to-noise (SNR) is lower than conventional BOTDRs. To overcome this weak point, we propose a coded S-BOTDR using a Golay code sequence. The coded S-BOTDR's spectrum agrees with that of S-BOTDR on average; hence the high spatial resolution feature of S-BOTDR is preserved. We show that the spectrum's SNR increased and that the number of repetitions can be reduced by a coded S-BOTDR, although a limit exists that cannot be exceeded by increasing the code length.
\end{abstract}

\section{Introduction}

Brillouin optical time-domain reflectometry (BOTDR) is a distributed sensing method of strain and temperature in an optical fiber based on the property in which the Brillouin spectrum shifts in proportion to the strain and temperature [1]. A conventional BOTDR injects a single pulse into an optical fiber and obtains the Brillouin spectrum by heterodyning the Brillouin backscattered light. Unfortunately, its spatial resolution has been limited to 1 meter [2], which is insufficient for many applications. To overcome this spatial resolution limit, the double-pulse BOTDR (DP-BOTDR) [3], the synthetic BOTDR (S-BOTDR) [4], and the phase shift pulse BOTDR (PSP-BOTDR) [5] have been proposed. Instead of a single pulse of a conventional BOTDR, the DP-BOTDR uses two short pulses, and both S-BOTDR and PSP-BOTDR use composite pulses composed of short and long pulses. With these BOTDRs, 10-cm spatial resolution is possible.

However, such high spatial resolution BOTDRs have a problem: the signal-to-noise ratio (SNR) of the Brillouin spectrum is lower than the conventional BOTDR and needs more repeated measurements. To solve it, coding is one desired and promising technique [6]. For conventional BOTDRs, such coding techniques as Golay coding and simplex coding have already been applied to improve SNRs $[7,8]$, although the spatial reso- lution was limited to 1 meter. For DP-BOTDRs, pulse compression using Barker codes [9] has been proposed. In this paper, we apply phase modulation by the Golay complementary sequence [10] to a S-BOTDR and show that the SNR is improved while maintaining high spatial resolution.

\section{S-BOTDR}

\subsection{S-BOTDR using cross spectrum}

A block diagram of S-BOTDR is given in Fig. 1, where four measurements with different probe lights were carried out.

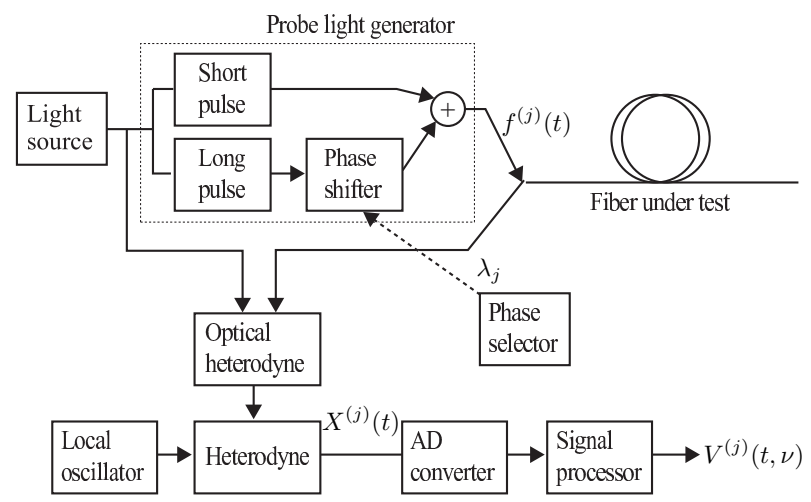

Fig. 1: Block diagram of S-BOTDR

The four probe lights are composed of short pulse $f_{1}(t)$ and long pulse $f_{2}(t)$ in Fig. 2 with the following different phase differences:

$$
f^{(j)}(t)=f_{1}(t)+r \lambda_{j} f_{2}(t), \quad j=1,2,3,4,
$$

where $r$ is the amplitude ratio between the short and long pulses and $\lambda_{j}, j=1,2,3,4$ are the phase factors that are selected to satisfy

$$
\left|\lambda_{j}\right|=1, \quad \sum_{j=1}^{4} \lambda_{j}=0, \quad \sum_{j=1}^{4} \lambda_{j}^{2}=0 .
$$

In what follows, $\lambda_{1}=1, \lambda_{2}=i, \lambda_{3}=-1, \lambda_{4}=-i$ are used to satisfy $(2)$.

Probe light $f^{(j)}(t)$ is injected into an optical fiber, and the Brillouin backscattered light is heterodyned with a reference light to yield heterodyned signal $X^{(j)}(t)$. 

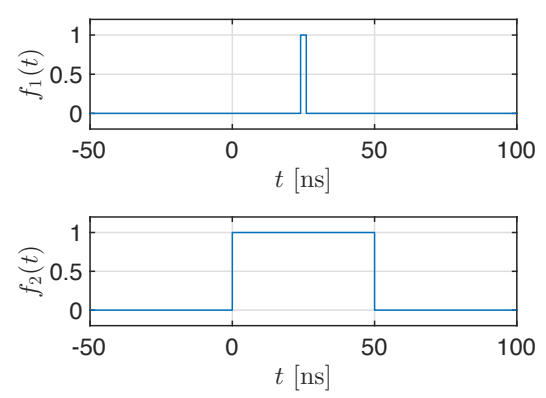

Fig. 2: Probe light elements of S-BOTDR

In a previous paper [4], a Brillouin spectrum was obtained by synthesizing four spectrums of $X^{(j)}(t), j=$ $1,2,3,4$. However, it is simpler to synthesize cross spectrums instead of the spectrums, as was previously adopted [5]. Since the final synthesized spectrum is the same, we use cross spectrums here.

The S-BOTDR procedure using cross spectrums is as follows:

1) Repeat 2)-7) $n_{\text {rep }}$ times.

2) Repeat 3)-5) for $j=1,2,3,4$.

3) Inject probe light $f^{(j)}(t)$ into an optical fiber and obtain heterodyned signal $X^{(j)}(t)$ from the backscattered light.

4) Extract the frequency components of $X^{(j)}(t)$ with frequency $\nu$ by two matched filters, $h_{1}(t)=$ $f_{1}(-t)$, and $h_{2}(t)=f_{2}(-t)$, which respectively correspond to short and long pulses:

$$
\begin{aligned}
& Y_{1}^{(j)}(t, \nu)=h_{1}(t) *\left[\exp (-2 \pi i \nu t) X^{(j)}(t),\right. \\
& Y_{2}^{(j)}(t, \nu)=h_{2}(t) *\left[\exp (-2 \pi i \nu t) X^{(j)}(t),\right.
\end{aligned}
$$

where $*$ denotes convolution.

5) Calculate the cross spectrum by

$$
V^{(j)}(t, \nu)=Y_{1}^{(j)}(t, \nu) \overline{Y_{2}^{(j)}(t, \nu)},
$$

where overline $\overline{\{\cdot\}}$ denotes the complex conjugate.

6) Calculate the synthetic spectrum by

$$
V_{\mathrm{S}}(t, \nu)=\Re\left(\sum_{j=1}^{4} \lambda_{j} V^{(j)}(t, \nu)\right) .
$$

7) Accumulate synthetic spectrum $V_{\mathrm{S}}(t, \nu)$.

8) For each time $t$, obtain the estimates of Brillouin frequency shift (BFS) $\nu_{\mathrm{B}}(z)$ at position $z=v_{\mathrm{g}} t / 2$ as the peak frequency of $V_{\mathrm{S}}(t, \nu)$, where $v_{\mathrm{g}}$ is the light velocity in an optical fiber. The peak frequency is obtained by parabola-fitting the logarithm of $V_{\mathrm{S}}(t, \nu)$.
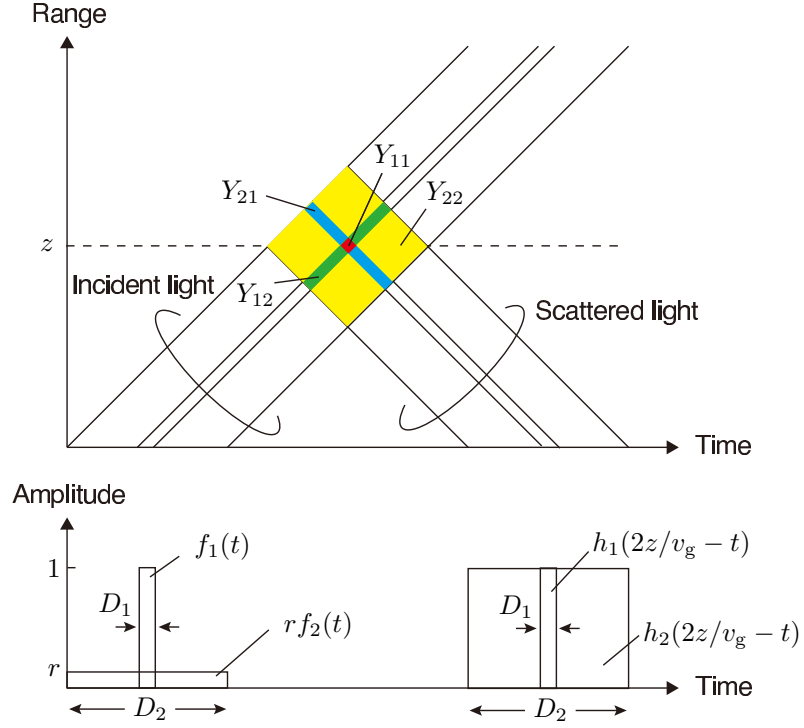

Fig. 3: Incident and scattered lights of S-BOTDR

\subsection{S-BOTDR performance}

For an arbitrarily fixed $\nu$, we represent the output from two matched filters (3) and (4) as follows:

$$
\begin{aligned}
& Y_{1}^{(j)}=Y_{11}+r \lambda_{j} Y_{21} \\
& Y_{2}^{(j)}=Y_{12}+r \lambda_{j} Y_{22}
\end{aligned}
$$

where $Y_{k l}, k, l=1,2$ on the right-hand side (RHS) represents the extracted frequency component of the scattered light from pulse $f_{k}(\cdot)$ by matched filter $h_{l}(\cdot)$. These correspond to the integral of the scattered lights in the different colored parts in Fig. 3. The real part of cross spectrum $Y_{11} \overline{Y_{22}}$ [4] becomes the ideal Brillouin spectrum if the pulse width of the short pulse is set to the desired spatial resolution and that of the long pulse is set to be sufficiently longer than the phonon lifetime. The cross spectrum for probe light $f^{(j)}$ is written as

$$
V^{(j)}=Y_{11} \overline{Y_{12}}+r \overline{\lambda_{j}} Y_{11} \overline{Y_{22}}+r \lambda_{j} Y_{21} \overline{Y_{12}}+r^{2} Y_{21} \overline{Y_{22}} .
$$

By taking the expectation of the synthetic spectrum, undesired terms are eliminated by condition (2) and yield

$$
E V_{\mathrm{S}}(t, \nu)=E\left(\sum_{j=1}^{4} \lambda_{j} V^{(j)}(t, \nu)\right)=4 r E\left(Y_{11} \overline{Y_{22}}\right) .
$$

The expectation can be approached by accumulating or averaging the synthetic spectrums obtained through many repeated measurements. However, since the peak power of the spectrum is low due to the nature of the high spatial resolution, the spectrum's relative fluctuation is larger than the conventional BOTDR and the number of repeated measurements increases. 


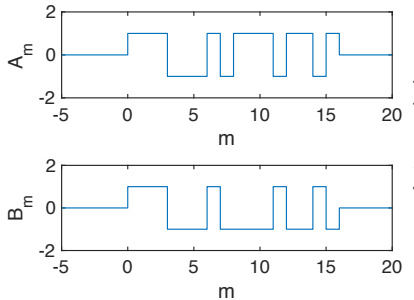

(a) Golay codes

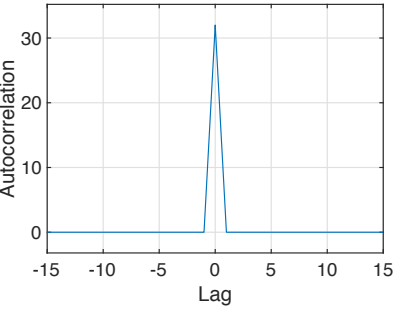

(b) Autocorrelation
Fig. 4: Example of Golay codes, $M=16$

\section{Golay-coded S-BOTDR}

To improve the S-BOTDR's SNR we utilize a coding technique by Golay code.

\subsection{Golay code sequence}

A pair of code sequences, $A_{k}, B_{k}, k=0,1, \cdots, M-1$, is called a complementary sequence if the sum of the autocorrelation satisfies

$$
\sum_{m=0}^{M-1}\left(A_{m} A_{m+k}+B_{m} B_{m+k}\right)=2 M \delta_{k, 0}
$$

where $\delta_{k, 0}$ is the Kronecker delta. Golay code is a binary complementary sequence taking values \pm 1 [10] that can be generated by the following appending method:

$$
\left\{\begin{array}{c}
A \\
B
\end{array}\right\} \rightarrow\left\{\begin{array}{c|c}
A & B \\
A & -B
\end{array}\right\}
$$

Here is an example:

$$
\begin{aligned}
& \left\{\begin{array}{l}
1 \\
1
\end{array}\right\} \rightarrow\left\{\begin{array}{rr}
1, & 1 \\
1, & -1
\end{array}\right\} \rightarrow\left\{\begin{array}{rrrr}
1, & 1, & 1, & -1 \\
1, & 1, & -1, & 1
\end{array}\right\}
\end{aligned}
$$

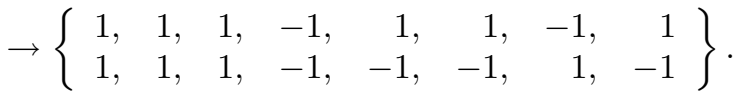

An example of a Golay code with a length of 16 and its autocorrelation is shown in Fig. 4. We code the probe pulses of the S-BOTDR using the Golay code.

\subsection{Construction of probe lights}

In an S-BOTDR, four composite pulses that combine short and long pulses are used as probe lights. For each composite pulse, two sequences of Golay code are generated; eight sequences are generated in total for eight probe lights.

For example, the following sequences are used in a
Golay code with $M=4$ :

$\begin{array}{rllrrrrr}A, \lambda_{1} & : & \{ & 1 & 1 & 1 & -1 & \} \\ A, \lambda_{2} & : & \{ & i & i & i & -i & \} \\ A, \lambda_{3} & : & \{ & -1 & -1 & -1 & 1 & \} \\ A, \lambda_{4} & : & \{ & -i & -i & -i & i & \} \\ B, \lambda_{1} & : & \{ & 1 & 1 & -1 & 1 & \} \\ B, \lambda_{2} & : & \{ & i & i & -i & i & \} \\ B, \lambda_{3} & : & \{ & -1 & -1 & 1 & -1 & \} \\ B, \lambda_{4} & : & \{ & -i & -i & i & -i & \}\end{array}$

Coding is applied to the phase of either short or long pulses. Let $d$ be the duration of the composite pulses, and let $M$ be the number of codes. The eight probe pulses are constructed as follows:

$$
\begin{aligned}
& f^{(A, j)}(t)=\sum_{m=0}^{M-1}\left[f_{1}(t-m d)+\lambda_{j} A_{m} r f_{2}(t-m d)\right], \\
& f^{(B, j)}(t)=\sum_{m=0}^{M-1}\left[f_{1}(t-m d)+\lambda_{j} B_{m} r f_{2}(t-m d)\right], \\
& j=1,2,3,4
\end{aligned}
$$

where superscripts $(A, j)$ and $(B, j)$ respectively stand for code sequences $\left(A, \lambda_{j}\right)$ and $\left(B, \lambda_{j}\right)$. Although the long pulses are coded in this case, the short pulses could be coded instead. Fig. 5 shows an example of a coded probe light and a scattered light of a coded S-BOTDR with $M=4$, where the short pulses are coded.

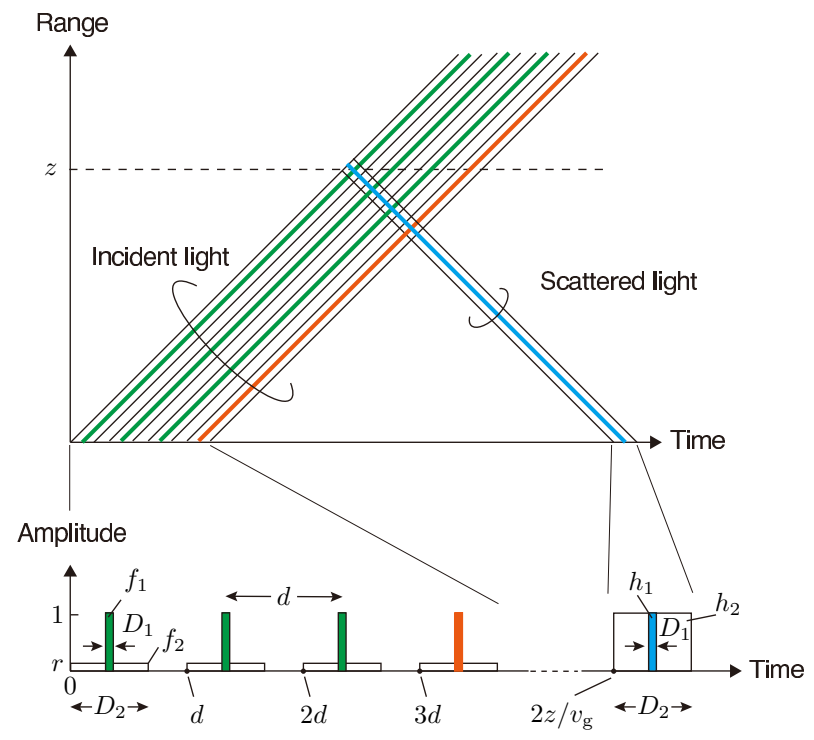

Fig. 5: Incident and scattered lights of Golaycoded S-BOTDR

\subsection{Signal processing of coded S-BOTDR}

Let $X^{(A, j)}(t), X^{(B, j)}(t) j=1,2,3,4$ be the heterodyned signals at time $t$, obtained by injecting probe lights $f^{(A, j)}(\cdot), f^{(B, j)}(\cdot), j=1,2,3,4$. The coded SBOTDR procedure with signal processing is as follows: 
1) Repeat 2)-8) $n_{\text {rep }}$ times.

2) Repeat 3)-5) for $j=1,2,3,4$.

3) Inject probe light $f^{(A, j)}(t)$ into an optical fiber and obtain heterodyned signal $X^{(A, j)}(t)$ from the backscattered light.

4) Extract the frequency components of $X^{(A, j)}(t)$ with frequency $\nu$ by two matched filters, $h_{1}(t)=$ $f_{1}(-t)$, and $h_{2}(t)=f_{2}(-t)$, which respectively correspond to short and long pulses:

$$
\begin{aligned}
& Y_{1}^{(A, j)}(t, \nu)=h_{1}(t) *\left[\exp (-2 \pi i \nu t) X^{(A, j)}(t)\right] \\
& Y_{2}^{(A, j)}(t, \nu)=h_{2}(t) *\left[\exp (-2 \pi i \nu t) X^{(A, j)}(t)\right] .
\end{aligned}
$$

5) Calculate the cross spectrum by

$$
V^{(A, j)}(t, \nu)=Y_{1}^{(A, j)}(t, \nu) \overline{Y_{2}^{(A, j)}(t, \nu)} .
$$

6) Calculate the synthetic spectrum with respect to $A$ by

$$
V_{\mathrm{S}}^{A}(t, \nu)=\sum_{j=1}^{4} \lambda_{j} V^{(A, j)}(t, \nu)
$$

7) Obtain $V_{\mathrm{S}}^{B}(t, \nu)$, which is the synthetic spectrum with respect to $B$, by the same procedure as 2$)-6$ ) for $A$.

8) Accumulate synthetic spectrums $V_{\mathrm{S}}^{A}(t, \nu)$ and $V_{\mathrm{S}}^{B}(t, \nu)$.

9) Decode the coded synthetic spectrums by

$$
\begin{aligned}
& V_{\mathrm{dec}, \mathrm{S}}(t, \nu) \\
& =\sum_{m=0}^{M-1}\left[A_{m} V_{\mathrm{S}}^{A}(t+m d, \nu)+B_{m} V_{\mathrm{S}}^{B}(t+m d, \nu)\right],
\end{aligned}
$$

taking the real part or the abstract value that yields the Brillouin spectrum.

10) For each time $t$, estimate $\nu_{\mathrm{B}}(z)$, which is the BFS at position $z=v_{\mathrm{g}} t / 2$, as the peak frequency of $V_{\mathrm{S}}(t, \nu)$. The peak frequency is obtained by parabola-fitting the logarithm of $V_{\mathrm{S}}(t, \nu)$.

\section{Performance analysis of Golay coded S-BOTDR}

\subsection{Expectation of decoded spectrum}

For arbitrarily fixed $\nu$, we represent (15) and (16), the output from two matched filters, as follows:

$$
\begin{aligned}
& Y_{1}^{(A, j)}=Y_{11}^{A}+r \lambda_{j} Y_{21}^{A}, \\
& Y_{2}^{(A, j)}=Y_{12}^{A}+r \lambda_{j} Y_{22}^{A},
\end{aligned}
$$

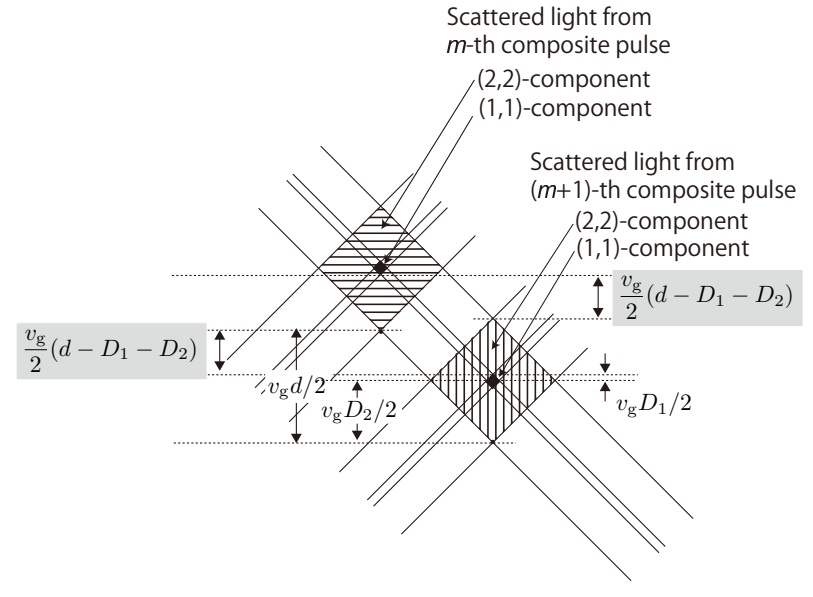

Fig. 6: Condition for distance between composite pulses of coded S-BOTDR

where $Y_{1 l}^{A}, l=1,2$ are the frequency components of the scattered light from the pulse train of short pulses $\sum_{m=0}^{M-1} f_{1}(t-m d)$ by matched filter $h_{l}(t)$ and $Y_{2 l}^{A}, l=1,2$ are from the pulse train of long pulses $\sum_{m=0}^{M-1} A_{m} f_{2}(t-m d)$. They are decomposed as

$$
\begin{aligned}
& Y_{1 l}^{A}(t, \nu)=\sum_{m=0}^{M-1} Y_{1 l}(t-m d, \nu), \\
& Y_{2 l}^{A}(t, \nu)=\sum_{m=0}^{M-1} A_{m} Y_{2 l}(t-m d, \nu) .
\end{aligned}
$$

By substituting (17), (20) and (21) into (18) and taking its expectation, undesired terms are eliminated, as previously done (10), yielding

$$
E V_{\mathrm{S}}^{A}=4 r E\left(Y_{11}^{A} \overline{Y_{22}^{A}}\right) .
$$

If we substitute (22) and (23) into (24), it includes double summation. For decoding by correlation, the double summation must be reduced to a single summation. Hence, the cross terms in the double summation must disappear:

$$
E Y_{11}(t-m d, \nu) \overline{Y_{22}\left(t-m^{\prime} d, \nu\right)}=0,
$$

must hold. This is equivalent to a condition where two regions, $Y_{11}(t-m d, \nu)$ and $Y_{22}(t-(m+1) d, \nu)$ in Fig. 6 , are separated in the spatial direction and yield

$$
d \geq D_{1}+D_{2} .
$$

We assume that this condition is satisfied. Then (24) becomes

$$
E V_{\mathrm{S}}^{A}=4 r \sum_{m=0}^{M-1} A_{m} E\left[Y_{11}(t-m d, \nu) \overline{Y_{22}(t-m d, \nu)}\right]
$$


Similarly, the synthetic spectrum with respect to $B$ sequence becomes

$$
E V_{\mathrm{S}}^{B}=4 r \sum_{m=0}^{M-1} B_{m} E\left[Y_{11}(t-m d, \nu) \overline{Y_{22}(t-m d, \nu)}\right] .
$$

Substituting (27) and (28) into the expectation of (19)) with property (11), we have

$$
E V_{\text {dec }, \mathrm{S}}(t, \nu)=8 \operatorname{MrE}\left(Y_{11} \overline{Y_{22}}\right),
$$

which we compare to (10) for the S-BOTDR, and the expectation of the spectrum becomes $2 M$ times larger than the S-BOTDR.

\subsection{Spectrum's SNR}

Since the intensity of the Brillouin backscattered light is a random variable, the Brillouin spectrum also becomes a random variable that fluctuates around its expectation. The factors that cause this fluctuation are the signal fluctuation and the receiver noise, both of which are independent factors. Let $P_{\text {peak }}$ be the expectation of the spectral peak, and let $\sigma_{\text {noise }}^{2}$ and $\sigma_{\text {sig }}^{2}$ be the variances of the fluctuations of the peak power caused by the noise and signal fluctuations for one measurement, respectively. We define the signal-to-noise ratio (SNR) for one measurement as

$$
\mathrm{SNR}_{1}=\frac{P_{\text {peak }}}{\sqrt{\sigma_{\text {sig }}^{2}+\sigma_{\text {noise }}^{2}}} .
$$

For $n$ repeated measurements, since $P_{\text {peak }}, \sigma_{\text {sig }}^{2}$, and $\sigma_{\text {noise }}^{2}$ all become $n$ times larger, the SNR is represented as

$$
\mathrm{SNR}_{n}=\sqrt{n} \mathrm{SNR}_{1} .
$$

For the coded Brillouin spectrums with code length $M$, while $P_{\text {peak }}$ and $\sigma_{\text {noise }}^{2}$ become $M$ times larger, $\sigma_{\text {sig }}^{2}$ becomes $M^{2}$ times larger than a non-coded Brillouin spectrum because each scattered light contains $M$ signals from different points (Fig. 5) and $M$ scattered lights are correlated at the decoded process. Therefore, the SNR of the coded S-BOTDR with code length $M$ becomes

$$
\operatorname{SNR}_{M, n}=\sqrt{n M} \frac{P_{\text {peak }}}{\sqrt{M \sigma_{\text {sig }}^{2}+\sigma_{\text {noise }}^{2}}},
$$

and

$$
\mathrm{SNR}_{M, n} \rightarrow \sqrt{n} \frac{P_{\text {peak }}}{\sigma_{\text {sig }}}, \quad(M \rightarrow \infty)
$$

which implies that the SNR increase has a limit.

\subsection{BFS estimation accuracy}

The relation between the estimation error for estimating BFS from the Brillouin spectrum and the SNR is given by [11]

$$
\sigma_{\nu_{\mathrm{B}}} \approx \frac{C}{\mathrm{SNR}},
$$

where $C$ is a constant that is determined by the factors except SNR. Although the denominator on the RHS is $\sqrt{\text { SNR }}[11]$ because an electrical SNR was used, the above equation holds for the optical SNR used in this paper.

In Fig. 7, the BFS estimation errors are plotted for a coded S-BOTDR obtained by substituting (32) into (34). Since the SNR for the coded S-BOTDR has a limit when $M$ becomes large, the BFS estimation error also has a lower limit, which is indicated by the curve labeled $M=\infty$ in Fig. 7 .

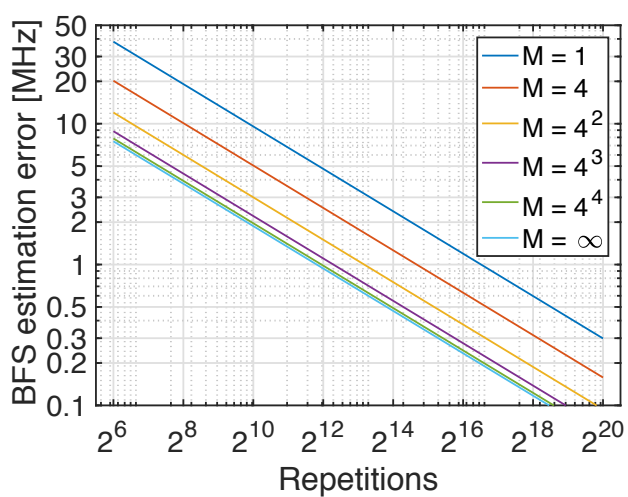

Fig. 7: Theoretical BFS estimation error

\section{Evaluation by simulation}

We performed a numerical simulation to verify the coded S-BOTDR and compared it with a non-coded S-BOTDR. The assumed BFS is given in Fig. 8.

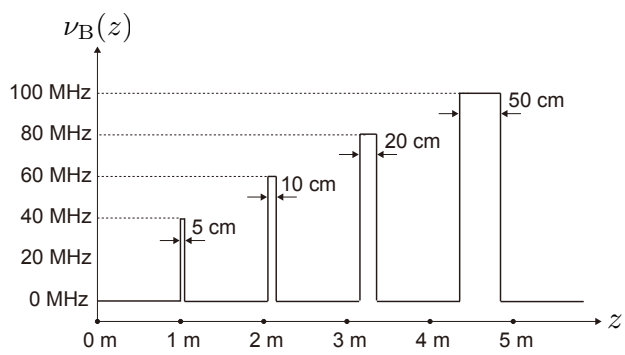

Fig. 8: Simulation condition: the vertical axis represents the distance from the input end of an optical fiber and the horizontal axis, the assumed BFS.

The pulse widths are $D_{1}=1 \mathrm{~ns}$ for a short pulse and $D_{2}=50 \mathrm{~ns}$ for a long pulse. The amplitude ratio is $\mathrm{r}$ $=0.08$. The short pulse is located at the center of the long pulse. The input power of the probe light is 20 $\mathrm{dBm}$. These parameters are shared by the S-BOTDR and the coded S-BOTDR.

The BFS estimation results for S-BOTDR are shown in Fig. 9. The number of repeated measurements is 


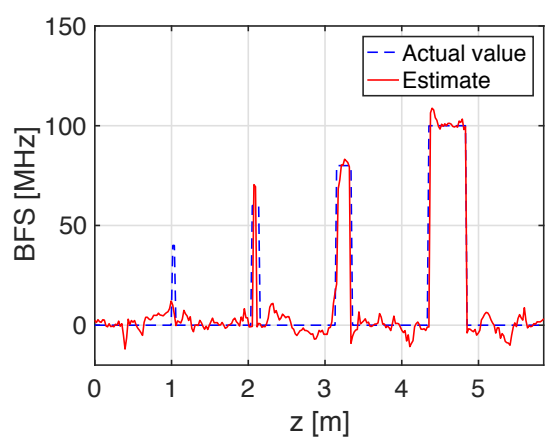

(a) $n_{\text {rep }}=2^{12}=4,096$

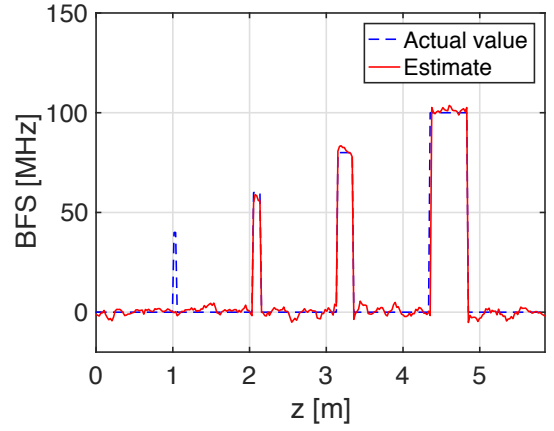

(b) $n_{\text {rep }}=2^{14}=16,384$

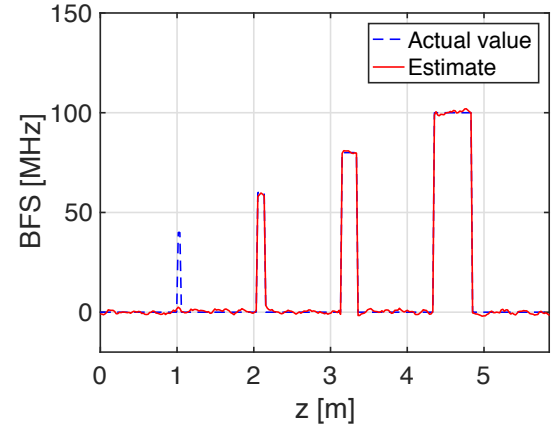

(c) $n_{\text {rep }}=2^{16}=65,536$

Fig. 9: BFS estimates by S-BOTDR

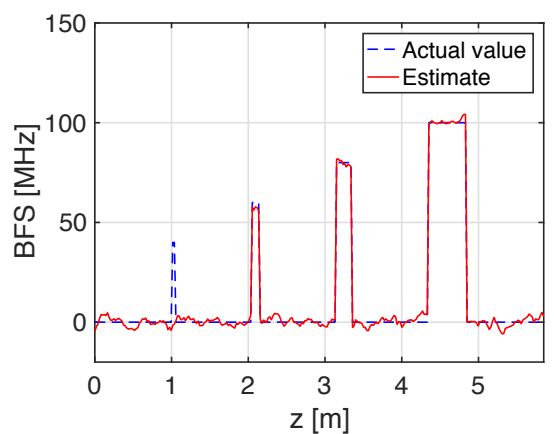

(a) $n_{\text {rep }}=2^{12}=4,096$

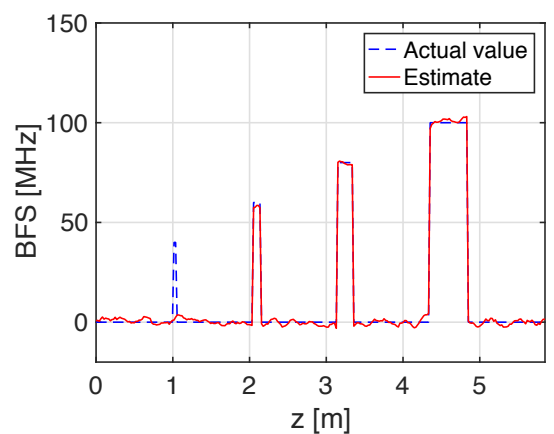

(b) $n_{\text {rep }}=2^{14}=16,384$

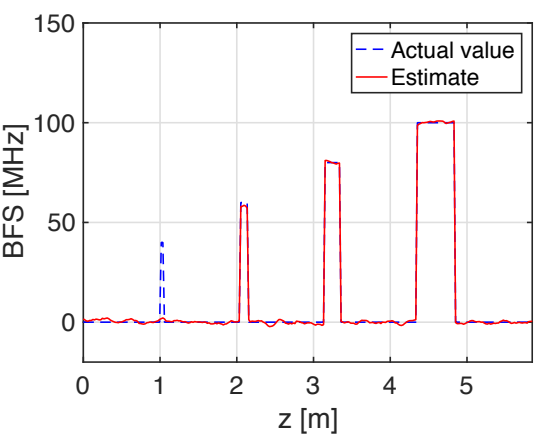

(c) $n_{\text {rep }}=2^{16}=65,536$

Fig. 10: BFS estimates by coded S-BOTDR, $M=4$

changed to $2^{12}, 2^{14}$, and $2^{16}$. Although no 5-cm intervals were detected in all cases since the pulse width of the short pulse is $1 \mathrm{~ns}$, which corresponds to $10-\mathrm{cm}$ spatial resolution, other intervals that equal or exceed 10 $\mathrm{cm}$ were all detected clearly, including edges. The estimation accuracy increases as the number of repetitions increases.

Figure 10 shows the BFS estimation results for a coded S-BOTDR with code length $M=4$. The number of repeated measurements is the same as for S-BOTDR in Fig. 9. From Figs. 9 and 10, we find that the BFS estimation accuracy of the coded S-BOTDR outperforms the S-BOTDR.

To quantitatively evaluate the BFS estimation accuracy, we calculated the root mean square (RMS) of the estimation errors in all the positions in a 15-m long uniform fiber. The RMS estimation error for the coded SBOTDR is plotted in Fig. 11. The curve labeled 'noise free' is the S-BOTDR simulation result without any receiver noise. It corresponds to a coded S-BOTDR with $M=\infty$ and becomes the substantial performance limit of the coded S-BOTDR. Although the simulation results of the coded S-BOTDR are only for the case of $M=4$, we expect to obtain closer performance by increasing the code length since there is a gap to the limit.

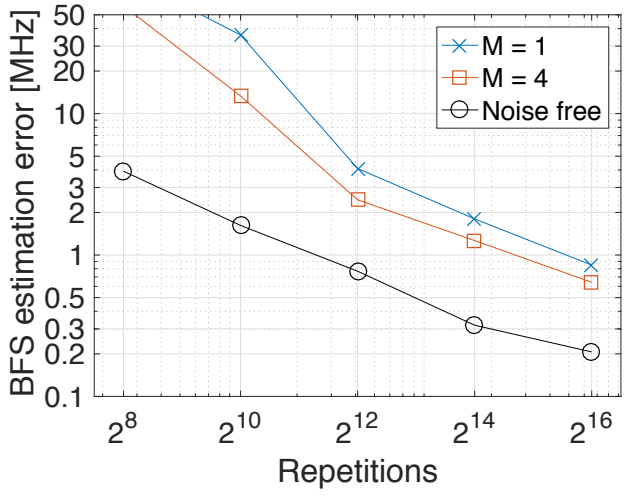

Fig. 11: BFS estimation error

\section{Conclusions}

A high spatial resolution BOTDR, such as S-BOTDR, has a weak point where the spectrum's SNR is lower than the conventional BOTDR. We proposed a coded S-BOTDR using a Golay code sequence to overcome this weak point. Since the spectrum of the coded SBOTDR basically agrees with that of the S-BOTDR, the high spatial resolution feature of S-BOTDR is preserved. The spectrum's SNR is increased, and the number of repetitions is reduced by the coded S-BOTDR. We also clarified that a limit exists that cannot be ex- 
ceeded by increasing the code length.

\section{Acknowledgement}

The author is grateful to Dr. Kinzo Kishida, Li CheHsien and Dr. Artur Guzik of Neubrex Co., Ltd. for their invaluable discussion and comments.

\section{References}

[1] T. Kurashima, T. Horiguchi, H. Izumita, S. Furukawa and Y. Koyamada, "Brillouin optical-fiber time domain reflectometry," IEICE Trans. Commun., vol. E76-B, no. 4, pp. 382-390, Apr. 1993.

[2] T. Kurashima, M. Tateda, T. Horiguchi, and Y. Koyamada, "Performance improvement of a combined OTDR for distributed strain and loss measurement by randomizing the reference light polarization state," IEEE Photon. Technol. Lett., vol. 9, no. 3, pp. 360-362, Mar. 1997.

[3] Y . Koyamada, Y . Sakairi, N. Takeuchi, and S. Adachi, "Novel technique to improve spatial resolution in Brillouin optical time-domain reflectometry," IEEE Photon. Technol. Lett., vol. 19, no. 23, pp. 1910-1912, Dec. 2007.

[4] K. Nishiguchi, Che-Hsien Li, A. Guzik, and K. Kishida, "Synthetic Spectrum Approach for Brillouin Optical Time-Domain Reflectometry," Sensors, vol. 14, no. 3, pp. 4731-4754, Mar. 2014.

[5] R. Shibata, H. Kasahara, and T. Horiguchi, "Proposal and demonstration of high spatial resolution BOTDR by correlating signals sampled with narrow- and wide-width window functions, " IEEE 6th International Conference on Photonics (ICP) 2016, Mar. 2016.

[6] T. Horiguchi, "Coding techniques for distributed fiber sensors based on Brillouin scattering," IEEE 6th International Conference on Photonics (ICP) 2016, Mar, 2016.

[7] M. A. Soto, G. Bolognini, and F. D. Pasquale, "Enhanced simultaneous distributed strain and temperature fiber sensor employing spontaneous Brillouin scattering and optical pulse coding," IEEE Photonics Technology Letters, vol. 21, no. 7, pp. 450-452, Apr., 2009.

[8] S. Wan, Y. Xiong, and X. He, "The theoretical analysis and design of coding BOTDR system with APD detector," IEEE Sensors J., vol. 14, no. 8, pp. 2626-2632, Aug. 2014.

[9] S. Matsuura, M. Kumoda, Y. Anzai, H. Akabane, and Y. Koyamada, "Enhancing performance of double-pulse BOTDR, " IEICE Trans. B, vol. J.97B, no. 10, pp. 962-972, Oct. 2014 (in Japanese).
[10] M. J. E. Golay, "Complementary series," IRE Trans. Inform. Theory IT-7, pp. 82-87, 1961.

[11] K. Nishiguchi, "Analysis of parameter estimation error for Brillouin distributed sensing," The 45th ISCIE International Symposium on Stochastic Systems Theory and Its Applications, pp. 325-330, Okinawa, Japan, Nov. 2013. 